\title{
Contributions of family physicians to health care services in Nigeria
}

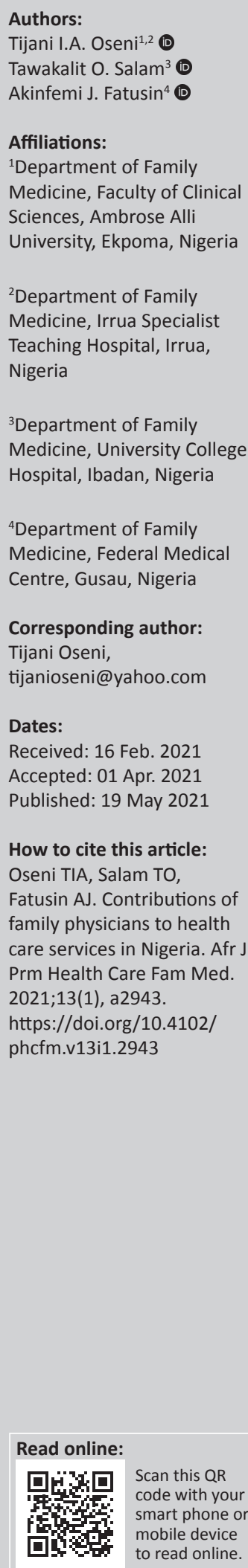

\begin{abstract}
In Nigeria, family physicians are doctors with specialised training to manage a broad range of clinical conditions and pathologies when they first present, considering the psychosocial, economic, cultural and environmental context of the individual and his or her family. In Nigeria, family physicians may be found at district hospitals but are more likely to be located at tertiary health care facilities, where their roles in medical education, research and clinical services cannot be overemphasised. Many patients present to tertiary facilities with primary-care problems, bypassing primary and secondary care. They are often seen initially by family physicians in general outpatient clinics, where $70 \%$ of all problems are managed without referral to other specialists. These physicians are also in charge of most of the National Health Insurance Scheme (NHIS) clinics nationwide. They are thus the gatekeepers to the majority of tertiary hospital services.
\end{abstract}

Keywords: family physicians; health care; general outpatient clinics; NHIS; contributions, Nigeria.

\section{Introduction}

Family physicians (FPs) in Nigeria provide patient-centred, comprehensive, coordinated, integrated, longitudinal and holistic care to patients irrespective of their age, gender or disease presentation, in the context of their family, culture and environment. ${ }^{1}$ They provide primary care to patients in primary health care (PHC) settings, district hospitals and specialist or teaching hospitals using the principles of PHC (equity, appropriate technology and selfreliance, community participation, intersectoral collaboration and integrated services). ${ }^{2}$

\section{Family physicians in district hospitals}

Although most doctors in district hospitals (private, central and general hospitals) do not have specialist training in family medicine, ${ }^{3}$ local governments and private hospitals across the country are increasingly seeing the need to employ FPs in these hospitals. Wherever available, FPs in these hospitals render highly specialised, cost-effective, integrated, coordinated and comprehensive care (inpatient, outpatient and surgeries) to patients irrespective of their disease presentation..$^{1,4}$ The relevance of FPs in district hospitals can be depicted by the clinical experience of one of the authors (Box 1).

Family medicine educators have also helped to improve the impact of family medicine in district hospitals through the introduction of a Diploma in Family Medicine programme by the National Postgraduate Medical College of Nigeria. ${ }^{5}$ A good number of general practitioners have obtained the Diploma in Family Medicine. This is expected to improve health care services and reduce unnecessary referrals from these hospitals to tertiary centres as well as decrease morbidity and mortality across the country.

\section{Family physicians in tertiary health care facilities}

Tertiary hospitals (national hospital, teaching hospitals, federal medical centres and specialist hospitals) are health care facilities where different specialists render clinical services. Tertiary facilities are referral centres for most district hospitals in Nigeria, especially for complicated cases. However, in Nigeria, many patients present to tertiary hospitals directly for primary care, mainly because of a dearth of primary- and secondary-care facilities as well as human resources for health.

\section{General outpatient clinics}

There are over 50 tertiary hospitals in the country, ${ }^{6}$ and these hospitals have general outpatient clinics (GOPC) staffed by FPs. These GOPCs serve as the gateway to the hospitals, as most patients coming to the hospitals pass through them. Family physicians provide first-contact comprehensive and coordinated care to these patients. They manage chronic diseases such as hypertension and 
BOX 1: Patient story from a district hospital.

A middle-aged male taxi driver presented with right groin swelling of four month ' duration. He had poor libido and was unable to conduct his business because of scrotal pain upon prolonged sitting. He was constrained financially and was having marital discord. The family physician (FP) seized the opportunity of the consultation to give comprehensive care to the patient and his family. He was diagnosed with a huge right inguinoscrotal hernia, type 2 diabetes mellitus and bilateral immature huge right inguinoscrotal hernia, type 2 diabetes melltus and bilateral immature cataracts. He had his blo sugar controlled and a successful surgery by the FP, who linked him to a nearby ophthalmologist. His wife, who was about 6 months pregnant, also received maternal care. The patient and his wife were satisfied with the care they received, and his quality of life improved. The FP was able to manage , as well as resolvinis patient and the maternal and child health needs of his family, as well as resolving the family dysfunction, in a holistic manner.

BOX 2: Patient story from a tertiary hospital.

The special role of a family physician (FP) in tertiary health care in Nigeria can b depicted in the care of B.O., a 65-year-old retired administrative officer, referred depicted in the care of B.O., a 65 -year-old retired administrative officer, referred hyperplasia and elevated prostate specific antigen, to rule out prostate cancer. There was a history of low back pain, generalised body weakness and weight loss There was a history of low back pain, generalised body weakness and weight loss of two years' duration, but no urinary symptoms. After further evaluation, the urologist determined that B.O. did not have prostate cancer but referred him to the orthopaedic surgeon on account of severe lumbar spondylosis. B.O.'s symptoms persisted despite treatment, so he defaulted from the clinic. He presented at the GOPCs about six months after trying different traditional, complementary, alternative medicines and spiritual options with no relief of symptoms. The FP evaluated B.O. and requested a full blood count, which revealed anaemia, and an erythrocytes sedimentation rate, which was markedly elevated. Further evaluation revealed a positive faecal occult blood test and metastatic bone lesions on a bone scan. He was referred to the gastroenterologist for colonoscopy, but no colorectal cancer was found. At this point B.O. continued to be seen by the FP. An abdominal computed tomoris to be

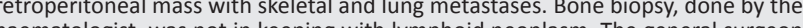
haematologist, was not in keeping with lymphoid neoplasm. The general surgeon retroperitoneal mass. The FP then linked B.O. with a palliative specialis. The FP played a major role in the coordination of care between multiple specialists in a tertiary health care facility. The FP was able to understand the patient's and family's perspective on the illness, including spiritual issues. The FP was trained to break bad news and understood the importance of ongoing palliative care.

diabetes and communicable diseases such as HIV, tuberculosis, neglected tropical diseases and outbreaks of emerging and reemerging infectious diseases such as Lassa fever, yellow fever and coronavirus disease 2019 (COVID-19). Over 70\% of these patients are treated solely by FPs, reducing costs as well as waiting times, as it could take up to three weeks to see other specialists from the time of referral. ${ }^{4}$ This also reduces the workload of other specialists, as the number of patients referred to them is significantly reduced. The way in which the FP can coordinate care within a tertiary hospital environment is illustrated in Box 2.

\section{National health insurance scheme}

Health expenditure in Africa is mainly out-of-pocket spending. ${ }^{7}$ In order to achieve universal health coverage, the National Health Insurance Scheme (NHIS) was introduced in Nigeria in 2005 in the formal sector. Most tertiary hospitals across the country have their NHIS clinics attached to family medicine clinicsand runby FPs. Thisis becauseFPsmanageundifferentiated illnesses irrespective of age, gender or disease entity. ${ }^{1}$ In addition, the wide scope of the discipline and the varied illnesses attended to make the services cost-effective to the NHIS. ${ }^{1}$

\section{Medical training and research}

Family physicians in tertiary hospitals also serve as researchers and educators/trainers of both undergraduates and postgraduates in Family Medicine. Acknowledging the significant contributions of family medicine to health care services in the country, the National Universities Commission and the Medical and Dental Council of Nigeria, which are charged with regulating university education and medical practice in the country, respectively, incorporated the teaching of family medicine into the undergraduate medical curriculum. ${ }^{8,9}$ This measure has ensured that nearly all medical graduates from Nigeria are taught the principles of family medicine in patient management. ${ }^{10}$

\section{Other contributions}

Family physicians have also strengthened the health system through their contributions to emergency, rehabilitative, geriatric and well-person care. They lead the accident and emergency units of some tertiary hospitals in the country. Their role in overseeing and coordinating the care of the elderly in the very few geriatric centres in the country cannot be overemphasised. ${ }^{11}$ The FP plays a key role in ensuring a multidisciplinary approach in care of the elderly, by coordinating other stakeholders involved in elder care, such as family members, nurses and other specialists. Despite these important roles, there has been a progressive decline in the numbers of FPs and trainees in Nigeria because of migration overseas.

\section{Conclusion}

The contributions of FPs to health services in Nigeria are invaluable. There is a need to train and retain FPs at all levels of health care in the country, including tertiary health institutions, where coordination of care and postgraduate medical training in Family Medicine are essential. Furthermore, there should be greater political will to engage the services of FPs at district hospitals and in support of PHC as we work towards universal health coverage.

\section{Acknowledgements} Competing interests

The authors declare that they have no financial or personal relationships that may have inappropriately influenced them in writing this article.

\section{Authors' contributions}

T.I.A.O. conceived the manuscript. T.I.A.O., T.O.S. and A.J.F. wrote the draft and edited and validated the final manuscript.

\section{Ethical considerations}

This article followed all ethical standards for research without direct contact with human or animal subjects.

\section{Funding information}

This research received no specific grant from any funding agency in the public, commercial or not-for-profit sectors.

\section{Data availability}

Data sharing is not applicable to this article as no new data were created or analysed in this study. 


\section{Disclaimer}

The views and opinions expressed in this article are those of the authors and do not necessarily reflect the official policy or position of any affiliated agency of the authors.

\section{References}

1. Jamoulle M, Resnick M, Vander Stichele R, Ittoo A, Cardillo E, Vanmeerbeek M Analysis of definitions of general practice, family medicine, and primary health care: A terminological analysis. BJGP Open. 2017;1(3):bjgpopen17X101049. https://doi.org/10.3399/bjgpopen17X101049

2. Oyedeji R, Abimbola S. How tertiary hospitals can strengthen primary health care in Nigeria. Niger Med J 2014;55(6):519-520. https://doi.org/10.4103/0300-1652.144715

3. Von Pressentin KB, Mash RJ, Baldwin-Ragaven L, Botha RPG, Govender I, Steinberg WJ. The bird's-eye perspective: How do district health managers experience the impact of family physicians within the South African district health system? A qualitative study.

4. Jimbo M. Family medicine: Its core principles and impact on patient care and medical education in the United States. Keio J Med. 2004;53(2):69-73. https:// doi.org/10.2302/kjm.53.69
5. Ajayi AO. The history of the Faculty of General Medical Practice, National Postgraduate Medical College of Nigeria (1980-2005). A paper delivered at the International Conference to mark the 25th Anniversary of the GMP Faculty; 2005 Nov 3 [cited 2021 Feb 8]. Available from: http://ifrs-rural.com/ HistoryofFamilyMedicineinNigeria.pdf

6. Healthcare resource guide: Nigeria. 2016 [cited 2021 Feb 14]. Available from: https://2016export.gov/industry/health/healthcareresourceguide/

7. Mash $\mathrm{R}$, Howe $\mathrm{A}$, Olayemi $\mathrm{O}$, et al. Reflections on family medicine and primary healthcare in sub-Saharan Africa. BMJ Glob Health. 2018;3(Suppl 3):e000662. https://doi.org/10.1136/bmjgh-2017-000662

8. Federal Ministry of Health of Nigeria, Health Systems 20/20 Project. Nigeria undergraduate medical and dental curriculum template, 2012. Bethesda, MD: Health Systems 20/20 Project, Abt Associates Inc.; 2012.

9. Medical and Dental Council of Nigeria. Guidelines on minimum standards of medical and dental education in Nigeria. The Red Book. Revised ed. Abuja: Medical and Dental Council of Nigeria; 2006.

10. Okokon IB, Ogbonna UK, Asibong UE, Aluka TM, Dienye PO. Teaching family medicine in medical schools: The perspective from a Nigerian Medical School. Nigerian J Fam Pract. 2012;2(2):1-6.

11. Nigeria Health Watch. Ageing but not infirm: Getting geriatric care right in Nigeria. 2018 [cited 2021 Mar 16]. Available from: https://nigeriahealthwatch.com/ ageing-but-not-infirm-getting-geriatric-care-right-in-nigeria/ 